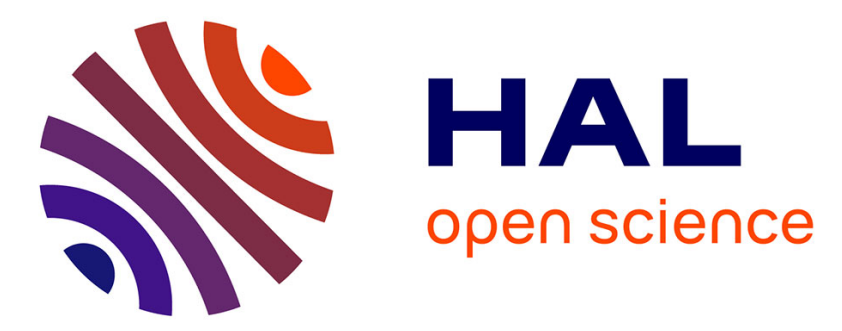

\title{
Cognition, énaction, perculturation : vers une caractérisation du lexique anglais dans une perspective typologique
}

Didier Bottineau

\section{- To cite this version:}

Didier Bottineau. Cognition, énaction, perculturation : vers une caractérisation du lexique anglais dans une perspective typologique. Langages, 2019, Le lexique et ses implications: entre typologie, cognition et culture, $\mathrm{N}^{\circ} 214$ (2), pp.91. 10.3917/lang.214.0091 . hal-03090900

\section{HAL Id: hal-03090900 https://hal.science/hal-03090900}

Submitted on 30 Dec 2020

HAL is a multi-disciplinary open access archive for the deposit and dissemination of scientific research documents, whether they are published or not. The documents may come from teaching and research institutions in France or abroad, or from public or private research centers.
L'archive ouverte pluridisciplinaire HAL, est destinée au dépôt et à la diffusion de documents scientifiques de niveau recherche, publiés ou non, émanant des établissements d'enseignement et de recherche français ou étrangers, des laboratoires publics ou privés. 


\author{
Didier Bottineau \\ CNRS, UMR 5191 ICAR, ENS de Lyon
}

\title{
Cognition, énaction, perculturation: vers une caractérisation du lexique anglais dans une perspective typologique
}

Obi-Wan Kenobi: I have a bad feeling about this.

Qui-Gon Jinn: I don't sense anything.

Obi-Wan: It's not about the mission, Master. It's something... elsewhere. Elusive.

Qui-Gon: Don't center on your anxieties, Obi-Wan. Keep your concentration here and now, where it belongs.

Obi-Wan: But Master Yoda said I should be mindful of the future.

Qui-Gon: But not at the expense of the moment. Be mindful of the living Force, young Padawan.

(Star Wars, Episode 1, The Phantom Menace, George Lucas, 1999)

\section{Problématique : mot-acte et « perculturation » dans le lexique anglais}

Dans son Introduction descriptive à la lexicogénétique de l'anglais contemporain, J. Tournier (1985 : 407 sqq.) mentionne « l'élément culturel » juste avant la conclusion (10.4.) : il n'en fait pas une force organisatrice du lexique mais reconnaît son importance dans une section documentée. Notre approche vise à montrer comment le fait culturel joue un rôle structurant pour le système actuel du lexique anglais compte tenu de ses sources diachroniques métissées, à l'origine de doublons de langue bien connus comme feel et sense (Allen, 1908, Walter, 2001, Crystal, 2003, Svartvik \& Leech, 2016, Sesterhenn, 2016), parfois à l'origine de doublets de discours, notamment dans la langue juridique (Heyden, 2012). Elle prend appui sur les principes de la linguistique énactive (de l'anglais enact et du français en acte "produire sur le scène théâtrale ": Maturana \& Varela, 1980 ; Bottineau, 2010, 2017ab). Poursuivant 1'analyse du langage comme energeia et activité (Aristote, 2000, W. von Humboldt, 1836/1974, E. Coseriu, 1968/2001), phénoménologiquement constituante de la réalité en tant qu'expérience vécue relative à l'activité incarnée et sociale propre à l'espèce humaine (Uexküll, 1934/2010, Sapir, 1983 et Whorf, 1939/1956, Merleau-Ponty, 1945, Benveniste, 1966), l'approche énactive envisage la parole comme éthologie constituante (le languaging, Maturana 1978) et technique vocale de conceptualisation qui produit le sens linguistique à la conscience des participants (parole intérieure réflexive et parole adressée communicationnelle). Selon l'énaction, la réalité vécue par les êtres vivants est une construction en devenir, dont le contenu est déterminé par un mode de production incarnée, luimême régulé par le mode de fonctionnement évolutif de l'espèce, son «domaine consensuel d'interactions » en devenir « autopoïétique ».

Pour sa part, l'humain a développé un sous-domaine comportemental, le languaging, qui lui permet à la fois d'orienter la manière dont l'expérience du réel est énactée et de synthétiser un monde mental idéel assumé comme distinct, le sens linguistique, assumé comme représentation et commentaire du «réel» où s'inscrit la parole. L'enjeu d'une linguistique énactive est de comprendre comment les pratiques langagières, dans leur dimension formelle (classes d'opérateurs, règles combinatoires) et corporelle (sensorimotricité du signifiant, de la prosodie, la gestualité, les mimiques, en situation et avec encadrement culturel et symbolique) contribuent à orienter la lecture du monde réel (telle que l'énaction générale le produit) et la constitution du sens linguistique dans sa relation à ce « réel ». Une langue forme un appareil interactionnel propre à une communauté, en auto-organisation continue (un système social dit "autopoiétique »: Luhmann, 1990) selon un processus de réplication de la relation interlocutive (Roulland, 2017). Sa pratique implique l'enculturation des individus qui s'adaptent à son environnement matériel et orientateur 
psychologiquement et intellectuellement (Meade 1963), leur « inverbation » somatique et psychique (Ladmiral \& Lipiansky, 1989 : 71 sqq.), l'émergence de modes d'existence psychosociale collectifs (Flamm 1990) dont l'explicitation normative tire un caractère national (Humboldt, 1822/2000). Dans ce cadre, le mot est défini comme un modèle d'acte moteur (Jousse, 2008 : 423 sqq. assimile la récitation à la manducation) dont les effets sensoriels activent des réminiscences conceptuelles et interactionnelles à la manière de la madeleine proustienne (Robert, 2003: 266), mais avec un contenu symbolique normatif: comme le souligne l'approche lexiculturelle en didactique des langues (Galisson, 1988, 1999; Lino \& Pruvost, 2003), le mot est un relais dialogique perculturant; chaque épisode d'une unité lexicale fait revivre à ses usagers un modèle de conceptualisation inscrit dans un modèle d'interaction avec toutes ses implications sociétales et culturelles.

Or le lexique anglais actuel présente de nombreux doublons comme liberty et freedom : il hybride deux répertoires anciens introduits par la rencontre de deux populations, germanique et romane, parlant des langues de types endocentrique et exocentrique (Baron \& Herslund, 2005) dont les types systémiques affectent les propriétés du lexique (Herslund, 2003, 2005). La collision et la fusion de ces deux systèmes, avec leurs divergences préalables, va se résoudre par la mise en place d'un système autopoïétique original, avec des redistributions systémiques et effets réplicatifs sur les populations amalgamées en partie hérités de l'impulsion initiale : la diachronie du système ne retient de l'apport historique que ce qui contribue à l'organiser en distinguant des processus cohérents (Begioni \& Rocchetti, 2015). Il en résulte un lexique interculturel diachroniquement métissé et en relation synchronique avec d'autres facteurs typologiques corrélatifs comme la syntaxe constructionnelle. On étudie la manière dont la collision de deux répertoires lexicaux se résout par un système intégré où se jouent deux modes de conceptualisation du sens (section 2) munis de propriétés de distributionnelles, morphologiques et sémantiques (section 3).

\section{Collision systémique et émergence de deux modes de conceptualisation interactive}

L'anglais est connu pour présenter de très nombreux doublons germaniques et romans tels que freedom et liberty, que l'on ne retrouve pas dans d'autres langues germaniques (allemand Freiheit) ou romanes (français liberté). Ces doublons se présentent dans tous les champs sémantiques: l'alimentation (sheep: mouton vivant, mutton: viande de mouton), les phénomènes naturels (wind/vent), les sensations (feel et sense 'sentir', 'ressentir'), l'action (deed et act 'action'), la cognition (aware et conscious 'conscient'), les rapports sociaux (fame et celebrity 'célébrité'). Le phénomène concerne des noms d'objets non dérivés (dress/robe, wire/cable; ground/soil, grass/herb, mind/spirit), des couples de noms simples ou dérivés (heed/attention), des verbes (give/donate, guess/ divine, breathe/respire, respirate 'faire respirer'), des adjectifs avec ou sans dérivation (huge/enormous, holy/saint), des notions lexicales transcatégorielles (buy/purchase, call/appeal). Les paires sont encore plus nombreuses si l'on prend en compte les termes obsolètes (king/roy) et les termes quasi-obsolètes qui ne se conservent que dans des emplois très spécialisés (hand/main au sens de cast of dice 'lancer de dés').

La signification de cette distribution pose problème et se prêterait facilement à des interprétations outrancières fondées sur les modes de vie et les mentalités: vu du XXI siècle, mutton au sens de "viande» semble suggérer que les Normands du XIe siècle, en situation patricienne, ne parlent que de l'aliment alors que sheep indiquerait que les Saxons en rôle plébéïen au service des Normands ne s'intéressent qu'à l'élevage. En réalité, en amont se sa mise en opposition à sheep, mutton désigne chez les Normands aussi bien le mouton que la viande (Burchfield, 1985 : 18, cf. let's return to our muttons), comme mouton en français actuel. C'est la rencontre des deux mots dans des interactions verbales croisées qui les force à se mettre en système d'opposition distinctive dont la valeur s'articule autour d'un contraste fondé sur des croyances stéréotypiques construites par chaque groupe relativement à l'autre et mutualisées au sein des actes de communication au point de former un clivage interculturel qui radicalise idéologiquement des 
faits historiques ténus, voire inexistants. La distinction semble s'installer au sein de textes médiateurs comme le Domesday Book (inventaire démographique, patrimonial et cadastral destiné à l'imposition, 1089), où mutton se focalise sur la viande et sheep sur l'animal, puis se propager dans l'usage (Kornexl \& Lenker, 2011) : elle reflète la coloration du sens lexical par des rôles sociaux pragmatiques et symboliques que les groupes en présence s'attribuent mutuellement au gré de rencontres et d'interactions verbales dans la durée historique plutôt que d'une différence linguistique préétablie fondée sur une réalité matérielle. Elle illustre l'émergence d'une mise en scène mythique et efficace, une énaction collective, sans motivation claire ni détermination contraignante, mais avec l'efficacité d'une vision commune à laquelle chacun peut souscrire comme à un pacte culturel qui s'ignore en tant que construction et précipite des effets de distinctions communautaires. Et surtout, notre hypothèse est que cette mise en système oppositif de sheep et mutton est emblématique d'un processus très général en anglais : la formation de doublons (et plus) comme microsystèmes hybrides multiples, constitutifs ensemble d'un macrosystème lexical intégrant, au sein duquel se produit un clivage sémantique interculturel qui affecte à la fois le signifié et les implications pragmatiques, interactionnelles et psychologiques prêtées aux sujets parlants à travers l'emploi des mots en contexte.

Ce clivage s'appuie sur l'écart typologique entre langues endocentriques et langues exocentriques (Baron \& Herslund, 2005) pour s'extrapoler en mobilisant des traits morphologiques et constructionnels corrélatifs qui lui permettent de s'affranchir de l'extraction saxonne ou normande des étymons recrutés, comme dans date/appointment ou arrest/detain, tous romans : le choix de date ou appointment distribue aux arguments et aux interlocuteurs des rôles sociaux symboliques inspirés par une motivation historique mais réorganisés en paires systémiques autonomes dont peut user tout locuteur selon l'interaction et les buts poursuivis. Le lexique anglais se clive en un système social auto-organisé et «autopoïétique » (Luhmann, 1990), impulsé par des facteurs historiques initiaux et ultérieurs (Guerre de Cent Ans, Renaissance, Lumières, révolution industrielle, Empire, mondialisation), et catalysé par la rencontre de paramétrages typologiques contrastés et évolutifs. Il témoigne d'une motivation non déterministe des circonstances historiques et linguistiques initiales, de leur appropriation symbolique par les populations qui les pratiquent et les observent, et de l'activation d'un principe systémique de distinction énactée au sein du lexique, lequel va œuvrer de manière autonome en diachronie à travers le languaging pour produire le système que l'on observe actuellement. Le clivage interculturel mis en place ne reflète pas des préjugés et stéréotypes initiaux, au contraire il résulte de la dynamique systémique même et va en s'amplifiant à la manière d'une rumeur ou d'une légende urbaine sans source localisable.

\section{Distribution systémique, formelle et sémantique du clivage}

Dans les doublons de type reach/attain, ache/pain, answer/reply, birthday/anniversary, le terme d'ascendance germanique inscrit le sens lexical dans un contexte interactionnel et pragmatique matériel et sensible, et implique les interlocuteurs comme collaborateurs engagés dans une tâche concrète de nature opérative : son emploi fait co-énacter le signifié en mode opératif, évoque le monde matériel, la posture actorielle, la perspective empirique et pragmatique, comme le « feeling » de l'apprenti Jedi Obi-Wan Kenobi dans la citation de Star Wars en exergue. Au contraire, le terme d'ascendance romane profile le sens lexical d'un point de vue théorique et abstrait, le fait coénacter en mode spéculatif, en tant qu'objet d'une discussion intellectuelle codifiée par des normes d'interaction verbales expertes, avec des interlocuteurs profilés comme co-producteurs d'un regard réflexif et distant sur une réalité transfigurée en univers symbolique, dématérialisé, intellectuel, socialement codifié, élitiste, comme le «sense » du maître Qui-Gon Jinn. En anglais actuel, la coexistence de ces deux modes offre au locuteur la possibilité de faire interpréter le référent via des notions lexicales qui font conceptualiser le signifié sous des angles différents selon le modèle d'interaction verbale convoqué par le terme choisi : chaque paire lexicale fonctionne comme un 
commutateur phénoménologique ancré dans des protocoles antagoniques de la co-construction du sens ; chaque sélection lexicale active un angle phénoménologique à l'exclusion de l'autre.

On distingue ainsi deux verbes «atteindre»: reach fait énacter un agent engagé dans un rapport corporel à un objet matériel dans une situation pratique, et fait énacter le couple interlocutif comme des collaborateurs verbaux eux-mêmes engagés dans une interaction pragmatique décontractée ; attain fait énacter un agent engagé dans un acte de pensée, l'idée d'atteindre par un geste mental un objet abstrait (attain a goal), tout en faisant énacter le couple interlocutif comme membres d'une élite engagés dans une interaction verbale intellectuelle et codifiée. De même, help fait énacter un agent matériel par des interlocuteurs " de seconde classe » en mode co-opératif alors que aid fait énacter un agent symbolique par des interlocuteurs " de première classe » en mode cospéculatif. La même personne peut dire lors d'une présentation powerpoint «We are not going to attain our objectives » ou en pause-café « We are not going to reach our objectives » selon la classe d'interaction qu'elle fait co-énacter entre elle-même et ses collègues, indépendamment de l'abstraction du référent. De ce fait, le lexique en mode spéculatif s'utilise dans des types de discours assumés comme techniques avec un locuteur ou rédacteur qui se pose en expert au sein d'une interaction verbale codifiée, alors que le lexique en mode opératif, ressenti comme plus spontané, se rencontre dans des types de discours, interactions et situations plus ordinaires, matérielles et pragmatiques. La distinction des deux modes lexicaux est dialogique, elle se fonde sur l'enregistrement du paradigme d'interactions au sein desquelles les mots s'incrustent usuellement.

Le mode opératif étant ancré dans l'action singulière, il tend à exprimer des situations spécifiques : oath of allegiance $=$ acte de serment d'allégeance au drapeau américain, au sein de l'armée et dans les écoles. Le mode spéculatif vise au général et peut former des hyperonymes : pledge of allegiance $=$ notion de serment d'allégeance en général. Un doublon lexical agit comme un commutateur phénoménologique : chacun de ses termes suscite un réseau complet d'implications théoriques et pratiques hétérogènes (cf. la notion de Culioli, 1999: 100, "système complexe de représentation structurant des propriétés physico-culturelles d'ordre cognitif »; la sémantique interprétative et l'herméneutique de Rastier, 1987, 1996), permettant au sujet parlant d'être préparé à des interfaces énactives complexes au moyen d'opérateurs lexicaux pré-adaptés simplexes (Berthoz, 2009), qui gèrent la complexité des mises en scène interactive sans la faire éprouver comme un problème à résoudre.

La distinction des modes opératif et spéculatif se projette aujourd'hui sur un lexique élargi, affranchi des doublons saxon/normand : on observe des doublons romans comme explain/explicate, efficient/efficacious, use/utilization avec la même valeur de commutateur phénoménologique, devenu autonome dans le système. On distingue explain 'expliquer' / explicate 'analyser' (un poème dans un exposé, rare et soutenu), parfois remplacé par explanate ( Bushism »), adjectif en biologie ( « de forme allongée ») et formation à rebours de explanatory. On oppose farming et grow (pratiques agricoles) à agriculture et cultivate (secteurs d'activité). Use (N/V) désigne l'usage ordinaire, utilize/utilization (of Arctic oil fields) l'exploitation d'une ressource. La répartition des termes est cohérente : le signifiant germanique, généralement monosyllabique, contraste souvent avec le signifiant roman par sa brièveté (send/envoy, grow/cultivate, own/possess, speech/discourse, scorn/disdain, staff/personnel, rainfall/precipitations, kernel/nucleus 'noyau' (de fruit ou d'atome), handle/manipulate, costly/expensive, cheap/economical, clever/intelligent, smart/astute). On trouve des paires de monosyllabiques (sell/vend, sing/chant, walk/march, help/aid, rope/cord (cf. vocal cords, the cord theory), heart/core (the earth's core), des paires de disyllabiques (outcome/result, aware/conscious), de trisyllabiques (sleeping-room/dormitory), de quadri-syllabiques (selfsufficient/autonomous), mais apparemment pas de doublon dont le terme spéculatif serait plus simple que le terme opératif. Le mode opératif a conservé de son origine germanique le modèle d'un signifiant compact, et le mode spéculatif, celui d'un signifiant manifestement sophistiqué, moins évocateur, propre à la mise en scène d'une interaction " patricienne » dans un registre plus élevé. On trouve bien des doublons de termes de complexité comparable, mais pas de doublons distribués «à contre-forme » : confrontés à ces paires, les locuteurs détectent l'effet différentiel du 
couplage et emploient les signifiants en fonction de leur complexité relative à l'autre terme, par un effet d'observation inconsciente et de mise en saillance. Le changement linguistique peut contribuer à accentuer l'écart sémiotique entre les signifiants par divers procédés tels que la diphtongaison et la troncation, qui rapprochent des mots romans du gabarit de type germanique, ou la surcomposition, qui rapproche tout mot du gabarit roman (cf. le Bushism misunderestimate) : destroy 'détruire' (vandalisme) vs. destruct 'procéder à la destruction' (d'un satellite en perdition avant sa rentrée dans l'atmosphère), avec deux termes romans qui reproduisent analogiquement le couple roman/germanique build vs. construct ( ${ }^{*}$ constroy, inattesté, énacterait une édification comme un acte de rebellion, tel un édifice illégal dans une Z.A.D.). Sur ce modèle avec signifiants romans simples et complexes, cf. improve/ameliorate, apply/applicate, imply/implicate, obvious/evident, fit/proper, suitable/appropriate.

Une autre manifestation du clivage simplicité/complication du signifiant s'observe dans les compositions : le mode opératif recueille des compositions transparentes typiquement germaniques (self-standing, à comparer à l'allemand selbstständig), et le mode spéculatif, des compositions de racines gréco-latines opaques (autonomous); cf. teenager/adolescent, child/infant, tiny/miniscule, user-friendly/ergonomic, eye doctor/ophthalmologist, understand/comprehend, usefulness/utility, waterfall/cascade, weathering/erosion, young/juvenile, thankfulness/gratitude, drunk/intoxicated, easy/facile, eatable/edible, comestible, famous/celebrious. La transparence du mode opératif passe également par un autre trait typologique germanique, les constructions en verbes à particule (phrasal verbs) d'origine prépositionnelle, comme give up/abandon, make up/fabricate, bring up/educate, do without/dispense with, carry out/accomplish, make up for/compensate, give money back/refund et reimburse, $\mathrm{V}+$ up/down (go up, climb down) vs. ascend, escalate/descend, et des listes entières sont consacrées à ces doublons très connus (Taillé, 1995 : 66 sqq. ; Hart, 2017).

De même, pour l'expression des déplacements spatiaux dans la typologie syntaxique de L. Talmy (2000 : 177 sqq.) et la typologie lexicale de I. Baron \& M. Herslund (2005), la construction à cadrage satellitaire lexicalise l'expérience sensorimotrice de la navigation dans l'espace (he walked/ran/sauntered into the room) et souligne l'engagement corporel dans l'environnement matériel, ce qui la rattache au mode opératif : elle fait imaginer l'expérience du déplacement. Par contraste, la construction à cadrage verbal rattache l'expression de la trajectoire au verbe (he entered the room), occultant l'expression du mode d'engagement locomoteur par l'agent du déplacement et plaçant le locuteur en situation d'observateur extérieur relatif au mouvement, ce qui la rattache au mode spectatoriel : elle témoigne du déplacement sans souligner son expérienciation.

La distinction des modes opératif et spéculatif sous-tend des pans entiers du lexique, aussi ne se réduit-elle pas toujours à deux mots de langue à l'exclusion de tout autre. De fait, on observe bien des couples robustes, comme ground et soil, town et city, trade et commerce, travel(ler) et voyage( $r$ ), switch et commute, thwart et frustrate, dive et plunge, top et summit, strength et force, smoke et fume, steam et vapor, show et exhibit, freedom et liberty ou oath et pledge 'serment'. Mais le principe n'est pas celui de la synonymie : changer de mode implique à la fois changer de manière d'imaginer le monde et s'inscrire dans le mode de pratiques et d'interactions verbales d'une autre communauté, ce qui peut produire des ruptures de symétrie liées à l'hétérogénéité des discours de spécialité. Par exemple, stream 'courant' (opératif) s'oppose à current en océanographie (Gulf Stream, ocean currents) mais à river en géographie continentale. Child (boy, girl) s'oppose à infant, mais childish (boyish, girlish) à puerile (behaviour) et infantile (disease) selon que l'on se situe en médecine, psychologie, sociologie. Selon les contextes, les acceptions de stress fluctuent et s'opposent à tension, pressure, constraint, accent. Cette polylectalité (coexistence de modes interactionnels distincts qui organisent les formes et leurs emplois, Berrendonner et al., 1983) suggère qu'on ne peut faire l'économie d'archi-sous-systèmes ancrés dans les discours: les contrastes se forment au sein de variétés d'interactions verbales, en contexte discursif spécialisé. Le verbe spread '(se) projeter dans toutes les directions', '(se) répandre', cf. Spread! (jargon militaire : « dispersez-vous »; jargon pornographique : sans équivalent français à ma connaissance), en mode opératif, contraste avec expand, extend, disperse, proliferate selon la nature du sujet et la spécialité 
du discours. Des mots d'emblée spécialisés échappent à cette variation et figent l'alternance au niveau de l'invariant de "langue », qui inclut en fait la spécialisation discursive : oath et pledge, freedom et liberty, behaviour et comportment (cf. ce titre d'un ouvrage collectif Existential and Atmospheric Feelings in Depressive Comportment). Dans certains domaines, l'opposition joue entre ensembles de termes : pour l'éducation, en mode opératif schooling, teaching, learning, training, coaching, et en mode spéculatif education, instruction, formation, apprenticeship, entrainment (effet d'entraînement en physique, biologie, météorologie, et en conversation : lexical entrainment, adoption mimétique des expressions de l'autre), sans que l'on puisse opposer terme à terme des mots individuels de chaque ensemble.

Le clivage lexical intervient sur plusieurs domaines sémantiques corrélatifs :

- La production d'un monde vécu comme sensible (mode opératif) ou intelligible (spéculatif) : freedom (liberté individuelle), liberty (collective, philosophique et politique), avec une opposition qui intensifie la dimension concrète soulignée par I. Baron (2009) pour frihed en danois ; wire (câble métallique manipulable, cf. wire service) vs. cable (idée de réseau, cf. cable $T V$ et wire cable, le concret spécifiant l'abstrait) ; sleeping beauty vs. dormant volcano/bank account. La production d'une idée du rapport soi-autrui en tant qu'agents collaboratifs ou penseurs qui co-construisent une idée concertée, avec des effets d'empathie prononcés pour le mode opératif et de détachement professionnel pour le mode spéculatif (cf. young/juvenile, du point de vue du sociologue, ou jobless/unemployed, grown-up/adult, look like/resemble, main/principal; cf. help vs. aid (humanitarian aid), choose (a wine) vs. select (an option).

- L'inscription dans des modèles dialogaux et discursifs à caractère ordinaire ou expert (look like/resemble).

L'usage du système par les sujets parlants illustre leur sensibilité, comme le révèlent la terminologie et la phraséologie : "noyau » se dit kernel ou stone pour le fruit vs nucleus pour l'atome ou le noyau terrestre, mais les informaticiens parlent de kernel pour le noyau d'un système d'exploitation, s'exprimant comme des techniciens en action sur un noyau concret (même virtuel) et non comme des physiciens qui se figurent une entité spéculative et intangible, théoriquement matérielle. Si la Statue of liberty de New-York illustre la liberté comme abstraction collective, philosophique et politique, la Statue of freedom de la coupole du Capitole à Washington illustre la liberté d'action centrée sur l'individu; remplacer le titre du film Cry Freedom (R. Attenborough, 1987, sur l'activiste anti-apartheid Steve Biko) par Cry Liberty aboutirait au non-sens.

Le clivage entre modes opératif et spéculatif n'est pas déterminé par les propriétés matérielles ou abstraites du référent : il concerne des objets (wire/cable), des objets immatériels (mist/brume) ou abstraits (shape/form, warp/deform, mind/spirit, power/energy, trend/tendency, slump/depression), des qualités (dark/somber, light/clear, moist ou damp/humid), des concepts (freedom/liberty, work/labour), des sensations (feel/sense, overwhelm/submerge), des attitudes (watch/observe, wonder/marvel), des émotions (wrath/fury), des actions (move/budge, meet/encounter, throttle/strangle et strangulate, scatter/disperse, sell/vend, send/envoy, sing/chant, swap/exchange, win/gain), des processus (swell/inflate, undulate), des situations (own/possess, open/overt), des affects (weak/feeble et debile, weary/fatigued, illness et sickness/disease), des appréciations (plain/simple, old/aged, stern/austere, stifflrigid), des jugements (seem/appear, huge 'qui m'apparaît énorme'/enormous 'hors norme' (du point de vue du spécialiste), doable 'que nous pouvons réaliser' et makeable 'que nous pouvons produire' vs. feasible 'réalisable en soi' (trois modalités du «faisable » dont deux opératives et une spéculative).

Le clivage entre les modes est plus ou moins profond selon les paires considérées. A minima, il fait jouer l'angle phénoménologique (sickness/disease) sans affecter la dénotation, auquel cas les termes demeurent commutables dans la plupart des contextes avec un jeu d'alternance intériorisé par la compétence du sujet. Si ce changement affecte les domaines de référence, la commutabilité peut être réduite (reach/attain) ou neutralisée (anniversary " commémoration annuelle publique », 
birthday « commémoration annuelle privée du jour de naissance); en ce cas il n'y a pas alternance chez le sujet, et la paire est un construit de second ordre, de niveau métalinguistique, fondé sur la prise en compte de la diachronie, des emprunts et de la comparaison avec d'autres langues (comme le français). Si le clivage modélise un principe d'organisation du lexique de la langue, il ne prétend pas refléter un modèle de la compétence cognitive intériorisé par chaque sujet parlant pour tout le lexique : la réalisation du système est plus hétérogène que cela.

\section{Conclusion}

L'anglais a développé un macro-système d'opposition lexicale où se joue un contraste imaginaire entre modes de vie, de pensée, d'action et d'interaction, inspiré de motivations historiques éparses et ténues, et précipité par la dynamique du languaging en tant que système social matérialisé par les interactions verbales. Il en résulte une analyse intégrée où le fait culturel et social est relié au fait cognitif plutôt que relégué à une pragmatique du discours qui interviendrait secondairement par rapport à un système abstrait. Ce processus illustre la nécessité d'extraire l'analyse linguistique des systèmes lexicaux de langue sur l'interactivité située tout en préservant l'autonomie du niveau linguistique compte tenu de sa capacité à se développer selon une orientation propre. Par ailleurs, l'intégration des critères culturels, symboliques et pragmatiques au sein même des oppositions lexicales de langue fournit un outil prévisionnel conséquent relatif aux conditions de réemploi des mots et de leurs effets émergents dans toute leur hétérogénéité, ce qui ne peut qu'aider des apprenants de l'anglais langue étrangère, souvent démunis face aux implications pratiques de l'hybridité lexicale.

\section{Bibliographie}

Allen E. A. (1908), « English Doublets », PMLA 23/2, 184-239.

ARISTOTE (2000), Métaphysique (Traduction du grec ancien par J. Tricot), Paris : Vrin.

BARON I. (2009), «Les notions de liberté et de démocratie au Danemark et en France: Valeurs universelles ou culturelles?», in I. Korzen \& C. Lavinio (éds), Lingue, culture e testi istituzionali, Florence : Franco Cesati, 231-252.

BARON I. \& HERSLUND M. (2005), "Langues endocentriques et langues exocentriques. Approche typologique du danois, du français et de l'anglais », in M. Herslund \& I. Baron (éds), Le génie de la langue française, Langue française 145, 35-53.

BEGIONI L. \& ROCCHETTI A. (2015), « Quelles perspectives psychomécaniques pour une systémique comparée des langues romanes », Studia Universitatis Babeș-Bolyai. Philologia 3, Cluj : Cluj University Press, 9-21.

BenVeniste E. (1966), Problèmes de linguistique générale, Paris : Gallimard, 63-74.

Berrendonner A., Le Guern M. \& Puech G. (1983), Principes de Grammaire Polylectale, Lyon : P.U.L.

Berthoz A. (2009), La Simplexité, Paris : Odile Jacob.

Berthoz A. \& Andrieu, B. (2011), Le Corps en Acte. Centenaire de Merleau-Ponty, Nancy: Presses universitaires de Nancy.

BOLINGER D. L. (1950), « Rime, assonance and morpheme analysis », Word 6 (2), 117-136.

BotTineau D. (2010), "Language and enaction », J. Stewart, O. Gapenne, E. Di Paolo (eds), Enaction: toward a new paradigm for cognitive science, Cambridge, Mass.: MIT, 267-306.

BotTINEAU D. (2017a), « Langagement (languaging), langage et énaction, a tale of two schools of scholars : un dialogue entre biologie et linguistique en construction », Signifiances (Signifying), 1, 1, 11-38. 
BotTineau D. (2017b), « Du languaging au sens linguistique », in D. Bottineau \& M. Grégoire (éds), Langage et énaction: corporéité, environnements, expériences, apprentissages, Intellectica 68, 19-67.

BURCHFIELD R. (1985/2002), The English Language, Oxford: Oxford University Press.

CARRIER H. (1992), Lexique de la culture pour l'analyse culturelle et l'inculturation, Tournai : Desclée.

CoseriU E. (1968/2001), L'homme et son langage, in H. Dupuy-Engelhardt, J.-P. Durafour \& F. Rastier (éds), L'Homme et son langage, Louvain - Paris : Peeters, 13-30.

Culioli A. (1990), Pour une linguistique de l'énonciation, Formalisation et opérations de repérage, 2, Gap : Ophrys.

FEnNell B. A. (2001), A History of English, A Sociolinguistic Approach, Oxford: Blackwell.

FLAMM A. (1990), L'analyse psychogrammaticale, Lausanne : Delachaux et Niestlé.

GALISSON R. (1999), « La pragmatique lexiculturelle pour accéder autrement, à une autre culture, par un autre lexique », Etudes de linguistique appliquée 116, 477-496.

HART C. (2017), The Ultimate Phrasal Verb Book, New York: Barron's.

HeRsLund M. (2003), «Pour une typologie lexicale », in M. Herslund (éd.), Aspects linguistiques de la traduction, Bordeaux : Presses Universitaires de Bordeaux.

HERSLUND M. (2005), "Lingue endocentriche e lingue esocentriche : aspetti storici del lessico", in I. Korzen \& P. d'Achille (éds), Tipologia linguistica e società, Florence : Franco Cesati, 19-30.

HEYDEN B. (2012), "D'une culture de common law à une culture civiliste : la traduction des doublets juridiques anglais en français », in C. Pagnoulle (éd.), Traduire les droits, Université de Liège.

HumbolDT W. VON (1822/2000), „Ueber den Nationalcharakter der Sprachen“, 1822/24, in D. Thouard (éd.), Sur le caractère national des langues et autres écrits sur le langage, Paris : Points.

HumBOLDT W. VON (1836/1974), «La différence de construction du langage dans l'humanité et l'influence qu'elle exerce sur le développement spirituel de l'espèce humaine ou Introduction à l'œuvre sur le kav ", in Introduction à l'œuvre sur le kavi et autres essais (Traduction de l'allemand par P. Caussat), Paris: Seuil, 143-419.

JOUSSE M. (2008), L'anthropologie du geste, Paris : Gallimard, TEL.

KORNEXL L. \& LENKER U. (2011), "Culinary and Other Pairs: Lexical Borrowing and Conceptual Differentiation in Early English Food Terminology", in Bauer R. \& Krischke U. (eds), More than Words: English Lexicography and Lexicology Past and Present. Essays Presented to Hans Sauer on the Occasion of his 65th Birthday, Frankfurt: Lang, 179-206.

LADMIRAL J.-R. \& LiPIANSKY E.-M. (1989), La Communication interculturelle, Paris : Armand Colin.

Lino M. T. \& Pruvost J. (éds) (2003), Mots et lexiculture, Hommage à Robert Galisson, Paris: Honoré Champion.

LuHMANN N. (1990), Essays on Self-Reference, New York: Columbia University Press.

MaturanA H. R. (1978), "Biology of language: The epistemology of reality », in G. Miller et E. Lenneberg (eds), Psychology and biology of language and thought: Essays in honor of Eric Lenneberg, New York: Academic Press, 27-64.

Maturana H. R. \& VARELA F. J. (1980), Autopoiesis and cognition: the realization of the living, Dordrecht: Reidel.

MEAD M. (1963), "Papers in Honor of Melville J. Herskovits: Socialization and Enculturation", Current Anthropology 4/2, 184-188.

Merleau-Ponty M. (1945), Phénoménologie de la perception, Paris, Gallimard.

RASTIER F. (1987), Sémantique interprétative, Paris : PUF.

RASTIER F. (1996), «Représentation ou interprétation ? - Une perspective herméneutique sur la médiation sémiotique ", in V. Rialle \& D. Fisette (éds), Penser l'esprit : des sciences de la cognition à une philosophie de l'esprit, Grenoble : Presses Universitaires de Grenoble, 219239. 
ROBERT S. (2003), «L'épaisseur du langage et la linéarité de l'énoncé: vers un modèle énonciatif de production ", in A. Ouattara (éd.), Parcours énonciatifs et parcours interprétatifs - Théories et application, Paris: Ophrys, 255-274.

Roulland D. (2017), «Langage et réplication », in D. Bottineau \& M. Grégoire (éds), Langage et énaction : corporéité, environnements, expériences, apprentissages, Intellectica 2017/2, 68, 69-97.

SAPIR E. (1983), "Language and environment", in D. G. Mandelbaum (ed.), Selected Writings of Edward Sapir in Language, Culture, and Personality, Berkeley and Los Angeles: University of California Press.

SESTERHENN K. E. (2016), An overview of the phenomenon of doublets in English, A Thesis Submitted to the Graduate Faculty of The University of Georgia in Partial Fulfillment of the Requirements for the Degree master of arts, Athens, Georgia.

SVARTVIK J. \& LeEch G. (2016), English - One Tongue, Many Voices, London: Palgrave Macmillan.

TAILlE M. (1995), Histoire de la langue anglaise, Paris : Colin.

TALMY L. (2000), Toward a cognitive semantics: Concept Structuring Systems 1, Cambridge, Mass.: MIT Press.

TOURNIER J. (1985/2007), Introduction descriptive à la lexicogénétique de l'anglais contemporain, Paris-Genève : Champion-Slatkine.

UEXKÜLL J. VON (1934/2010), A foray into the world of animals and humans; with a theory of meaning, Minneapolis: University of Minnesota Press.

WALTER H. (2001), Honni soit qui mal y pense, Paris : Robert Laffont.

WHORF B. L. (1939/1956), "The relation of habitual thought and behavior to language", in Carroll J. B. (ed.), Language, Thought, and Reality: Selected Writings of Benjamin Lee Whorf, Cambridge, Mass.: MIT Press, 134-159. 